\title{
Application of the Prunus spp. cyanide seed defense system onto wheat: Reduced insect feeding and field growth tests
}

\section{Supporting Information}

Carlos A. Mora ${ }^{1+\hbar}$, Jonas G. Halter ${ }^{1,+}$, Cornel Adler ${ }^{2}$, Andreas Hund $^{3}$, Heidrun Anders ${ }^{2}$, Kang Yu ${ }^{3}$, Wendelin J. Stark ${ }^{1}$

${ }^{1}$ Department of Chemistry and Applied Biosciences, Institute for Chemical and Bioengineering, ETH Zurich, Vladimir-Prelog-Weg 1, 8093 Zurich, Switzerland

${ }^{2}$ Julius Kühn-Institut, Federal Research Centre for Cultivated Plants, Institute for Ecological Chemistry, Plant Analysis and Stored Product Protection, Königin-Luise-Str. 19, 14195 Berlin, Germany

${ }^{3}$ Department of Environmental Systems Science, Institute of Agricultural Sciences, ETH Zurich, Universitätsstrasse 2, 8092 Zurich, Switzerland

‡These authors contributed equally.

*Email: wendelin.stark@chem.ethz.ch, Phone: +41 44632 o9 8o, Fax : +41 446331083 


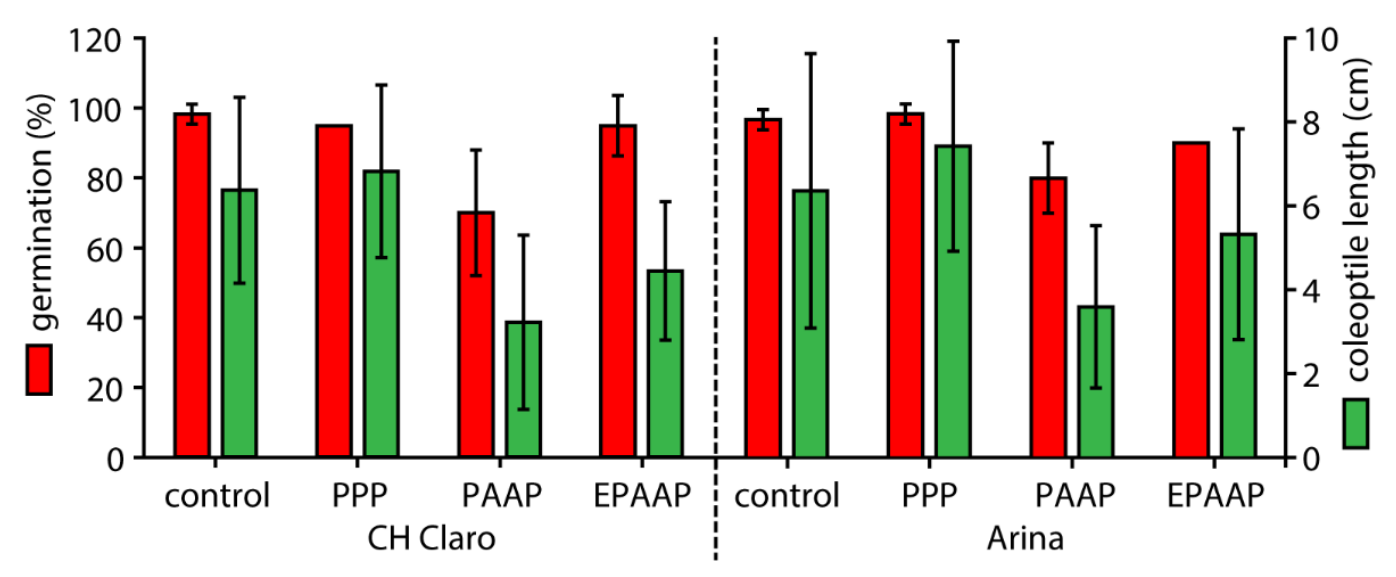

Figure S1. Standard germination experiment after one-year storage at ambient temperature, $40 \%$ humidity of coated wheat grains of the winter wheat varieties ' $\mathrm{CH}$ Claro' and 'Arina' (Delley Samen und Pflanzen AG, Switzerland). Coleoptile lengths (red), 7 days after sowing, and germination ability (green) of coated and uncoated grains. 


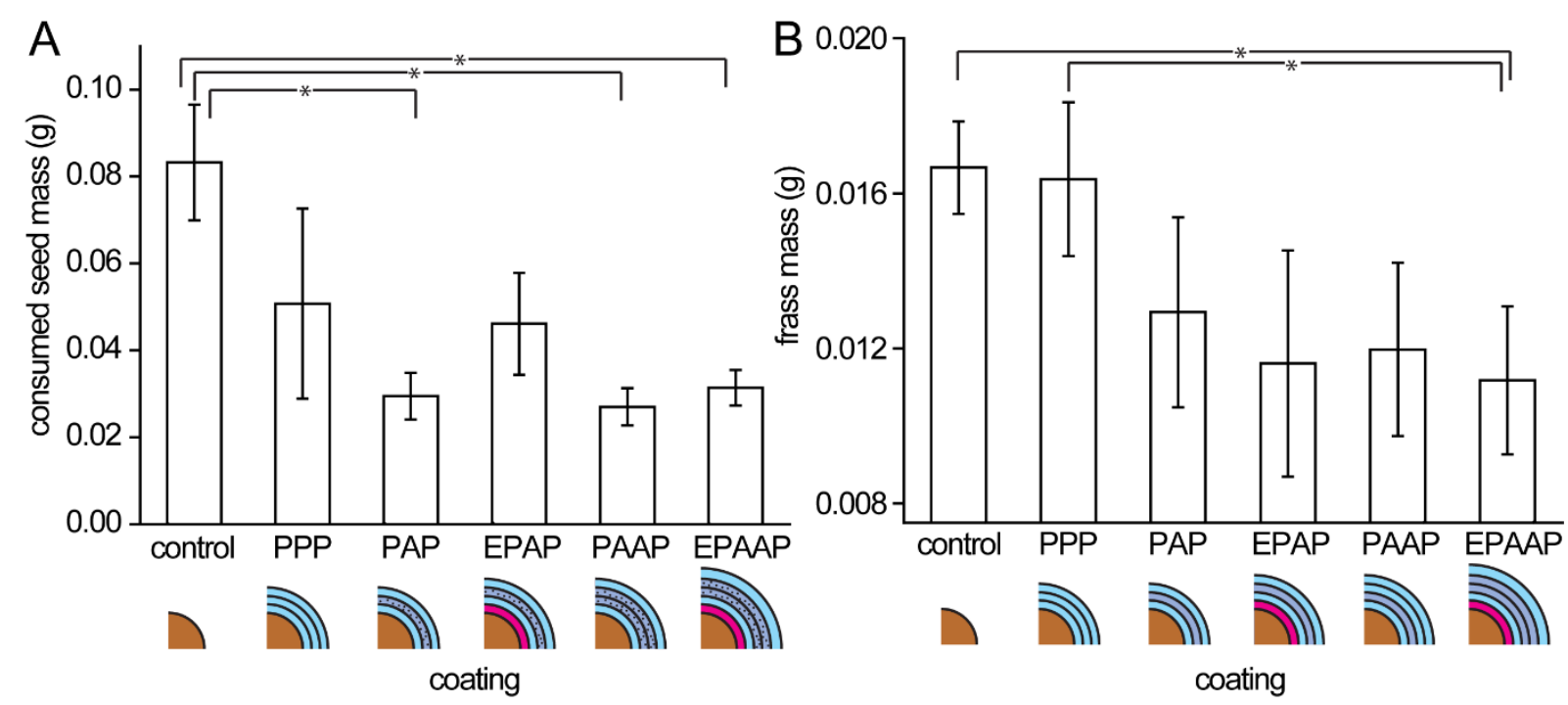

Figure S2. Effect of cyanogenic grain coating on larvae of Tenebrio molitor (meal beetle) evaluated in a 5-day feeding experiment. (A) Consumed grain mass, i.e. the difference between grain mass before and after the experiment. The initial grain mass was 2 g. (B) Frass mass collected at the end of the experiment. 
Table S1. Calculated values of the HCN measurements in mmol $\mathrm{HCN}$ per $\mathrm{kg}$ grain of all wheat grain varieties with different coatings used in this study. Wheat type A: Wheat grains purchased from Morga, Switzerland (Demeter wheat, product \#6809). Wheat type B: Winter wheat variety 'CH Claro' (Delley Samen und Pflanzen AG, Switzerland). Wheat type C: Winter wheat variety 'Arina' (Delley Samen und Pflanzen AG, Switzerland).

\section{Wheat types}
Coatings
$\mathrm{A} / \mathrm{mmol} \mathrm{kg}^{-1}$
$\mathrm{B} / \mathrm{mmol} \mathrm{kg}^{-1}$
$\mathrm{C} / \mathrm{mmol} \mathrm{kg}^{-1}$

\begin{tabular}{llll}
\hline PAP & $0.53 \pm 0.26$ & a & a \\
EPAP & $1.16 \pm 0.39$ & a & a \\
PAAP & $1.84 \pm 0.71$ & $1.9 \pm 0.2$ & $1.9 \pm 0.2$ \\
EPAAP & $2.63 \pm 0.62$ & $2.7 \pm 0.6$ & $3.5 \pm 0.5$ \\
\hline
\end{tabular}

${ }^{\mathrm{a}}$ not prepared 
Table S2. (Upper part) Calculated values of the $\mathrm{HCN}$ measurements in mmol $\mathrm{HCN}$ per $\mathrm{kg}$ grain and mmol $\mathrm{HCN}$ per $\mathrm{kg}$ coating. Wheat grains purchased from Morga, Switzerland (Demeter wheat, product \#6809). (Lower part) Literature values with HCN threshold

\section{Coating} HCN conc. per grain mass

HCN conc. per coating mass

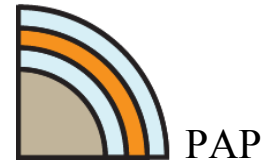

$$
0.53 \pm 0.26 \mathrm{mmol} \mathrm{kg}^{-1} \quad 15.3 \pm 7.6 \mathrm{mmol} \mathrm{kg}^{-1}
$$

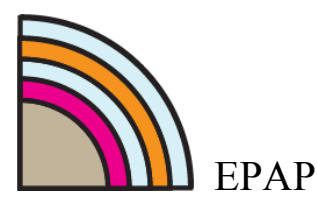

$$
1.16 \pm 0.39 \mathrm{mmol} \mathrm{kg}^{-1} \quad 33.3 \pm 11.3 \mathrm{mmol} \mathrm{kg}^{-1}
$$

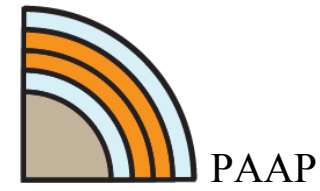

$$
1.84 \pm 0.71 \mathrm{mmol} \mathrm{kg}^{-1} \quad 53.0 \pm 20.3 \mathrm{mmol} \mathrm{kg}^{-1}
$$

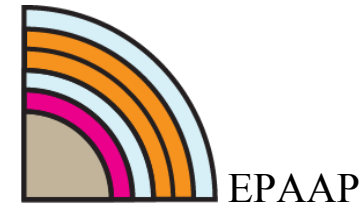

$$
2.63 \pm 0.62 \mathrm{mmol} \mathrm{kg}^{-1} \quad 75.6 \pm 17.8 \mathrm{mmol} \mathrm{kg}^{-1}
$$

Reference

Threshold concentration Effect

(in fresh biomass)

\begin{tabular}{lll}
\hline Patton, $1997^{1}$ & $2.4 \mathrm{mmol} \mathrm{kg}^{-1}$ & Dropped feeding intensity \\
Riis, $2003^{2}$ & $8.82 \mathrm{mmol} \mathrm{kg}^{-1}$ (a) & Reduced oviposition \\
Ballhorn, $2005^{3}$ & $15 \mathrm{mmol} \mathrm{kg}^{-1}$ & Repellent effect \\
Ballhorn, 2009 & $5-37 \mathrm{mmol} \mathrm{kg}^{-1}$ & Selective eating \\
\hline
\end{tabular}

concentrations having an effect on herbivores.

${ }^{(a)}$ Calculated from ppm values.

${ }^{1}$ Patton, C. A.; Ranney, T. G.; Burton, J. D.; Walgenbach, J. F. Natural pest resistance of Prunus taxa to feeding by adult Japanese beetles: Role of endogenous allelochemicals in host plant resistance. $J$. Am. Soc. Hortic. Sci. 1997, 122, 668-672.

${ }^{2}$ Riis, L.; Bellotti, A. C.; Bonierbale, M.; O'Brien, G. M. Cyanogenic potential in cassava and its influence on a generalist insect herbivore Cyrtomenus bergi (Hemiptera: Cydnidae). J. Econ. Entomol. 2003, 96, 1905-1914. 
${ }^{3}$ Ballhorn, D. J.; Lieberei, R.; Ganzhorn, J. U. Plant cyanogenesis of Phaseolus lunatus and its relevance for herbivore-plant interaction: The importance of quantitative data. J. Chem. Ecol. 2005, $31,1445-1473$.

${ }^{4}$ Ballhorn, D. J.; Kautz, S.; Heil, M.; Hegeman, A. D. Cyanogenesis of wild lima bean (Phaseolus lunatus L.) is an efficient direct defence in nature. Plos One 2009, 4, e5450.

Table S3. Cost analysis of coated grains in comparison to the cost of uncoated grains.

Uncoated wheat grains have a cost of $\sim 900 \$^{\star}$ per ton. ${ }^{a}$ This corresponds to $\sim 0.9 \$ \mathrm{~kg}^{-1}$.

Reading examples: Coated grains would therefore cost $0.95-1.05 \$ \mathrm{~kg}^{-1}$ for PAP and 0.96 -

$1.08 \$ \mathrm{~kg}^{-1}$ for EPAP coating which is $6-20 \%$ more expensive than the uncoated grains and $0.98-1.13 \$ \mathrm{~kg}^{-1}$ for PAAP and $0.98-1.16 \$ \mathrm{~kg}^{-1}$ for EPAAP coating which is $9-29 \%$ more expensive than the uncoated grains.

\begin{tabular}{|c|c|c|c|c|c|c|c|c|}
\hline \multirow[t]{2}{*}{ material } & \multirow[t]{2}{*}{$\begin{array}{l}\text { lab-scale } \\
\text { price }\end{array}$} & \multirow{2}{*}{$\begin{array}{l}\text { large- } \\
\text { scale } \\
\text { price }\end{array}$} & \multicolumn{2}{|c|}{$\begin{array}{l}\text { mass needed to } \\
\text { coat } 1 \mathrm{~kg} \text { of grain }\end{array}$} & \multicolumn{4}{|c|}{ cost per kg grain (large-scale) } \\
\hline & & & $\begin{array}{l}\text { PAP } \\
\text { and } \\
\text { EPAP }\end{array}$ & $\begin{array}{l}\text { PAAP } \\
\text { and } \\
\text { EPAAP }\end{array}$ & PAP & EPAP & PAAP & EPAAP \\
\hline PLA & $\begin{array}{l}291 \$ / \\
10 \mathrm{~g}^{\mathrm{b}}\end{array}$ & $\begin{array}{l}1.1-2.2 \$ \\
/ 1 \mathrm{~kg}^{\mathrm{c}}\end{array}$ & $44 \mathrm{~g}$ & $59 \mathrm{~g}$ & $\begin{array}{l}0.048- \\
0.097 \\
\$\end{array}$ & $\begin{array}{l}0.048- \\
0.097 \$\end{array}$ & $\begin{array}{l}0.065- \\
0.13 \$\end{array}$ & $\begin{array}{l}0.065- \\
0.13 \$\end{array}$ \\
\hline amygdalin & $\begin{array}{l}336 \$^{\ddagger} / \\
25 \mathrm{~g}^{\mathrm{d}}\end{array}$ & $\begin{array}{l}1-10 \$ / \\
1 \mathrm{~kg}^{\mathrm{e}}\end{array}$ & $5 \mathrm{~g}$ & $10 \mathrm{~g}$ & $\begin{array}{l}0.005- \\
0.05 \$\end{array}$ & $\begin{array}{l}0.005- \\
0.05 \$\end{array}$ & $\begin{array}{l}0.01- \\
0.1 \$\end{array}$ & $\begin{array}{l}0.01- \\
0.1 \$\end{array}$ \\
\hline$\beta$-Glucosidase & $\begin{array}{l}101 \$^{\ddagger} / \\
250 \mathrm{mg} \\
(\geq 6 \mathrm{U} / \mathrm{mg})^{\mathrm{f}}\end{array}$ & $\begin{array}{l}5-100 \$ / \\
1 \mathrm{~kg}^{\mathrm{g}}\end{array}$ & & $320 \mathrm{mg}^{\mathrm{h}}$ & - & $\begin{array}{l}0.0016- \\
0.032 \$\end{array}$ & - & $\begin{array}{l}0.0016- \\
0.032 \$\end{array}$ \\
\hline total coating & & & & & $\begin{array}{l}0.053- \\
0.15 \$\end{array}$ & $\begin{array}{l}0.055- \\
0.18 \$\end{array}$ & $\begin{array}{l}0.075- \\
0.23 \$\end{array}$ & $\begin{array}{l}0.077- \\
0.26 \$\end{array}$ \\
\hline
\end{tabular}

Calculated from $€$ or CHF $(1 €=1.09 \$ ; 1 \mathrm{CHF}=1.01 \$, 22.12 .2015)$

${ }^{a}$ Bioland Markt GmbH \& Co. KG, catalogue for spring 2016)

${ }^{\mathrm{b}}$ Polysciences, Inc. (http://www.polysciences.com/default/polyl-lactic-acid-mw-1600-2400,

22.12.2015)

${ }^{\mathrm{c}}$ Present price and forecast predicted by K.M. Nampoothiri et al. ${ }^{1}$

${ }^{\mathrm{d}}$ Sigma-Aldrich (http://www.sigmaaldrich.com/catalog/product/sigma/10050?lang=de\&region=CH, 22.12.2015)

${ }^{\mathrm{e}}$ Alibaba (http://www.alibaba.com/product-detail/Bitter-Apricot-Seed-Extract-

Amygdalin_742910688.html, 22.12.2015)

${ }^{\mathrm{f}}$ Sigma-Aldrich (http://www.sigmaaldrich.com/catalog/product/sigma/49290?lang=de\&region=CH, 22.12.2015)

${ }^{\mathrm{g}}$ Estimation made by Hensing et al. ${ }^{2}$

${ }^{\mathrm{h}}$ Activation was estimated to be 5 Units/mg (Brenda-Enzymes, EC 3.2.1.21)

${ }^{1}$ Nampoothiri, K. M.; Nair, N. R.; John, R. P., An overview of the recent developments in polylactide (PLA) research. Bioresource Technology 2010, 101, 8493-8501. 
${ }^{2}$ Hensing, M. C. M.; Rouwenhorst, R. J.; Heijnen, J. J.; Vandijken, J. P.; Pronk, J. T., Physiological and technological aspects of large-scale heterologous-protein production with yeast. Antonie Van Leeuwenhoek International Journal of General and Molecular Microbiology 1995, 67, 261-279. 